\title{
TAGUNG
}

\section{Die deutsche EU-Ratspräsidentenschaft - Herausforderungen und Erwartungen}

\author{
Jasmin Gassen*
}

Vom 1. Januar 2007 bis zum 30. Juni 2007 übernimmt die deutsche Bundesregierung die EU-Ratspräsidentschaft, für die sie das Motto „Europa gelingt gemeinsam“ wählte. In die Zeit der deutschen Präsidentschaft fällt das 50jährige Jubiläum der Gründung der EWG, die mit der Berliner Erklärung gewürdigt und zum Anlass des Rückblicks und der Vorausschau auf den europäischen Integrationsprozess genutzt wird. Neben den spezifischen Aufgaben in einzelnen Politikbereichen steht die EU vor der Herausforderung, ihre Zukunftsfähigkeit zu gestalten und in diesem Zusammenhang die Legitimität des europäischen Einigungsprozesses sowie dessen Akzeptanz in der europäischen Bevölkerung zu erhöhen. Welche Prioritäten die Bundesregierung zu diesem Zweck setzt und setzen sollte diskutierten Referenten und Teilnehmer auf einer vom Institut für Europäische Politik mit Unterstützung des Auswärtigen Amts, der EU-Kommission und der ASKO EUROPA-STIFTUNG in Zusammenarbeit mit dem EU-CONSENT Exzellenznetzwerk und TEPSA veranstalteten internationalen Fachtagung in Berlin. Die Konferenz ist Bestandteil einer Veranstaltungsreihe, die halbjährlich von den in der Trans European Policy Studies Association (TEPSA) zusammengeschlossenen außen- und europapolitischen Instituten der 27 EU-Mitgliedstaaten zur jeweils bevorstehenden Ratspräsidentschaft organisiert wird.

Staatssekretär Silberberg führte in das Tagungsthema ein, indem er zunächst die Rahmenbedingungen skizzierte, unter denen die deutsche EU-Ratspräsidentschaft agieren wird. Der ins Stocken geratene Verfassungs-

\section{Die EU voran bringen: Prioritäten der deutschen Ratspräsidentschaft}

Internationale Fachkonferenz des Instituts für Europäische Politik in Zusammenarbeit mit dem EU-CON-

SENT Network of Excellence und der Trans European Policy Studies Association sowie mit freundlicher Unterstützung des Auswärtigen Amtes, der EU-Kommission und der ASKO EUROPA-STIFTUNG

Berlin, 30. November / 1. Dezember 2006, Europasaal, Auswärtiges Amt

Begrüßung und Einführung: Reinhard SILBERBERG, Staatssekretär, Auswärtiges Amt, Berlin; Dr. Mathias JOPP, Direktor, Institut für Europäische Politik, Berlin; Prof. Dr. Wolfgang WESSELS, Jean-MonnetLehrstuhl, Universität zu Köln; Vorsitzender der Trans European Policy Studies Association (TEPSA), Brüssel; Koordinator des EU-CONSENT Network of Excellence

Eröffnungspanel: Prioritäten der deutschen Ratspräsidentschaft

Vorsitz: Dr. Mathias JOPP

Eröffnungsvortrag: Reinhard SILBERBERG

Kommentare: Andrew DUFF MdEP, Europäisches Parlament, Brüssel; Dr. Andres KASEKAMP, Direktor, Estonian Foreign Policy Institute (EVI), Tallinn; Dr. Petr DRULÁK, Präsident, Institute of International Relations, Prag; Horst BACIA, Korrespondent, Frankfurter Allgemeine Zeitung, Brüssel

Die Europäische Union zwischen Vertiefung und Erweiterung: Herausforderungen für die deutsche Präsidentschaft

Vorsitz: Dr. Barbara LIPPERT, Stellv. Direktorin, Institut für Europäische Politik, Berlin

Einführung: Peter TEMPEL, Ministerialdirigent, Leiter der Europaabteilung, Auswärtiges Amt, Berlin

Kommentare: Prof. Dr. Iain BEGG, European Institute, London School of Economics and Political Science, London; Michael STÜBGEN MdB, Europapolitischer Sprecher der CDU/CSU-Fraktion des Deutschen Bundestages, Berlin; Prof. Dr. José Ignacio TORREBLANCA, UNED Universität, Madrid; Real Instituto Elcano, Madrid; Prof. Dr. Maria KARASINSKA-FENDLER, European Institute, Lodz; Gaetane RICARD-NIHOUL, Generalsekretärin, Notre Europe, Paris

\footnotetext{
* Jasmin Gassen, Institut für Europäische Politik, Berlin.
} 
prozess, die zunehmende Europaskepsis sowie die aufgrund bevorstehender oder gerade durchgeführter Wahlen unklare innenpolitische Lage in einigen Mitgliedstaaten schränken Handlungsmöglichkeiten und Erfolgschancen ein. $\mathrm{Zu}$ den Rahmenbedingungen zählte Silberberg auch die sich verschiebenden Kräfteverhältnisse in der Weltpolitik, in der China, Indien und Russland (neue) Stärke gewinnen. Als wichtigste Eckdaten der Ratspräsidentschaft nannte Silberberg den Frühjahrsgipfel 2007, die Berliner Erklärung zum 50. Jahrestag der Römischen Verträge sowie den Europäischen Rat in Brüssel im Juni 2007. Auf der Tagesordnung des Frühjahrsgipfel am 8./9. März 2007 stehen die Lissabon-Strategie, Bürokratieabbau und die Stärkung von Forschung und Innovation, vor allem aber die EU-Energiepolitik. Ziel sei es, einen europäischen Aktionsplan für eine sichere und angesichts der Bedrohungen für das Weltklima eine umweltverträgliche Energieversorgung zu verabschieden. Die EU müsse energiepolitische Partnerschaften im Rahmen einer Energieaußenpolitik eingehen.

Die Berliner Erklärung zum 50. Jahrestag der Römischen Verträge am 25. März 2007 sei der Versuch eines Blicks nach vorn und solle von den Zielen und Werten der EU handeln. Sie solle als Impuls genutzt werden, den Bürgerinnen und Bürgern zu vermitteln, dass die europäische Integration dazu dient, die Herausforderungen des 21. Jahrhunderts $\mathrm{zu}$ bewältigen und die gemeinsame Zukunft $\mathrm{zu}$ gestalten. Die Intensivierung der Zusammenarbeit in der Innen- und Justizpolitik nannte Silberberg als Thema des Europäischen Rates im Juni 2007 in Brüssel. Hierzu zählt der weitere Ausbau der polizeilichen Zusammenarbeit, Europol und des Sicherheitsinformationssystems SIS II. Zudem wachsen die Herausforderungen durch einen steigenden Immigrationsdruck. Wichtige außenpolitische Herausforderungen stellen die Beziehungen zu Russland sowie die Einbeziehung Zentralasiens dar. In der Frage der Weiterführung des Verfassungsprozesses betonte Silberberg als Ziel aller weiteren Schritte das
Die Sicherheit der Bürger in der EU - Leistungsmöglichkeiten der europäischen Innen- und Justizpolitik (einschließlich Einwanderungspolitik)

Vorsitz: Brendan DONNELLY, Direktor, Federal Trust for Education and Research, London

Einführung: Peter ALTMAIER MdB, Parlamentarischer Staatssekretär beim Bundesminister des Innern, Berlin

Kommentare: Dr. Paul LUIF, Österreichisches Institut für Internationale Politik (OIIP), Wien; Christian AHRENDT MdB, Mitglied des Ausschusses für die Angelegenheiten der Europäischen Union, Deutscher Bundestag, Berlin; Prof. Dr. Siegfried MAGIERA, Deutsche Hochschule für Verwaltungswissenschaften Speyer

Gemeinsame Außen- und Sicherheitspolitik in einer unsicheren Welt (einschließlich EnergieauBenpolitik)

Vorsitz: Dr. Elfriede REGELSBERGER, Stellv. Direktorin, Institut für Europäische Politik, Berlin

Einführungen: Dr. Christoph HEUSGEN, Ministerialdirektor, Außen- und Sicherheitspolitischer Berater der Bundeskanzlerin; Leiter der Abteilung AuBen-, Sicherheits- und Entwicklungspolitik, Bundeskanzleramt, Berlin; Dr. Michael SCHAEFER, Ministerialdirektor, Politischer Direktor, Auswärtiges Amt, Berlin

Panelstatements: Prof. Dr. Geoffrey EDWARDS, Universität Cambridge; Dr. Hanna OJANEN, Finnish Institute of International Affairs, Helsinki; Graham AVERY, Senior Member of St. Antony's College, Oxford; Ehrengeneraldirektor, Europäische Kommission, Brüssel

Die soziale Dimension der EU und die LissabonStrategie

Vorsitz: Prof. Dr. Iain BEGG

Einführungen: Dr. Joachim WUERMELING, Staatssekretär, Bundesministerium für Wirtschaft und Technologie, Berlin; Kajo WASSERHÖVEL, Staatssekretär, Bundesministerium für Arbeit und Soziales, Berlin

Panelstatements: Dr. Angelica SCHWALL-DÜREN MdB, Stellv. Vorsitzende der SPD-Fraktion des Deutschen Bundestages, Berlin; Willem NOË, Vertretung der Europäischen Kommission in der Bundesrepublik Deutschland, Berlin; Dr. Ognian HISHOW, Forschungsgruppe EU-Integration, Stiftung Wissenschaft und Politik, Berlin

Impulse für eine neue Nachbarschaftspolitik der EU

Vorsitz: Dr. Gunilla HEROLF, Swedish Institute of International Affairs, Stockholm

Einführungen: Ekkehard BROSE, Ministerialdirigent, Beauftragter für EU-Außenbeziehungen und die Beziehungen zu den Mitgliedstaaten, Auswärtiges Amt, Berlin; Rutger WISSELS, Direktor Koordination Europäische Nachbarschaftspolitik, Europäische Kommission, Brüssel 
Festhalten an der politischen Substanz des Verfassungsvertrages (VVE), da der Vertrag von Nizza keine ausreichende Grundlage für eine funktionsfähige EU darstelle.

Der britische Europaabgeordnete Andrew Duff ergänzte den Themenkatalog um die $\mathrm{Zu}$ kunft des Balkans und die Statusklärung für das Kosovo, den NATO-Einsatz in Afghanistan und die Risiken für die transatlantische Partnerschaft. Duff empfahl, in der zweiten Hälfte 2007 eine kurze Regierungskonferenz mit einer nicht zu weit gefassten Agenda einzuberufen. Die Präambel sowie die Teile I und II des VVE sollten von den weiteren Verhandlungen unberührt bleiben! ${ }^{1}$

Andres Kasekamp verwies aus estnischer Perspektive darauf, dass es auch weiterhin bedeutend sei, im Rahmen der EU die Demokratisierungsprozesse in Osteuropa zu fördern und somit zur Stabilität in der Region beizutragen. Wichtig sei die Gestaltung der Beziehungen zwischen Russland und der EU, bei der alle Mitglieder beteiligt werden sollten. Petr Drulák führte aus, dass in Anbetracht der aktuellen Schwierigkeiten die Ratspräsidentschaft die Qualitäten eines ehrlichen Maklers benötigte. Da Deutschland über diese Qualitäten verfüge, werde es diese Rolle voraussichtlich gut ausfüllen und sollte nationale Sensibilitäten berücksichtigen. Horst Bacia bezweifelte, dass es möglich sei bei der Stabilisierung des Kosovo eine Lösung zu finden, die für beide Seiten akzeptabel sei. Hier sah er die Gefahr einer aufgezwungenen Lösung für eine Seite. Weiter sei eine stärkere Zusammenarbeit der EU und der NATO in Afghanistan nötig.

\section{Die Europäische Union zwischen Vertiefung und Erweiterung}

In einem Panel unter dem Vorsitz von Barbara Lippert zum Thema „Die Europäische Union zwischen Vertiefung und Erweiterung: Herausforderungen für die deutsche Präsident-
Panelstatements: Dr. Ettore GRECO, Visiting Fellow, Center on the United States and Europe, The Brookings Institution; Stellv. Direktor, Istituto Affari Internazionali, Rome; Dr. Atis LEJINS, Direktor, Latvian Institute of International Affairs, Riga; Krassimir NIKOLOV, Generalsekretär, Bulgarian European Community Studies Association (BECSA), Sofia; Rainder STEENBLOCK MdB, Europapolitischer Sprecher der Fraktion Bündnis 90/Die Grünen des Deutschen Bundestages, Berlin

\section{Abschlusspanel: Die Zukunft Europas}

Vorsitz: Prof. Dr. Christian FRANCK, Université Catholique, Louvain-la-Neuve; Generalsekretär, Trans European Policy Studies Association (TEPSA), Brüssel

Panelstatements: Axel SCHÄFER MdB, Europapolitischer Sprecher der SPD-Fraktion des Deutschen Bundestages, Berlin; Prof. Dr. Lenka ROVNA, Jean Monnet-Lehrstuhl ad personam, Karls-Universität, Prag; Prof. Dr. Jaap DE ZWAAN, Direktor, Netherlands Institute of International Relations Clingendael, Den Haag; Prof. Dr. Álvaro DE VASCONCELOS, Direktor, Instituto de Estudos Estrategicos e Internacionais (IEEI), Lissabon; Prof. Dr. Marjan SVETLICIC, Centre of International Relations, Ljubljana

Schlusswort: Prof. Dr. Wolfgang WESSELS

schaft" betonte Peter Tempel, Leiter der Europaabteilung im Auswärtigen Amt, dass die EU durch die Erweiterung nicht schwächer, sondern stärker geworden sei. Dennoch müsse die EU-Erweiterung mit Rücksicht auf die Aufnahmefähigkeit und Integrationsfähigkeit erfolgen, um auch eine große EU weiterhin handlungsfähig auszugestalten. Bedeutend im Hinblick auf die Funktionsfähigkeit der EU sei es, den europäischen Verfassungsprozess zu einem erfolgreichen Ende zu bringen. Hierfür müssten sich einige Mitgliedstaaten mehr bewegen als andere. In absehbarer Zeit stehe die Gestaltung der Vertiefung im Vordergrund, wobei aber die Erweiterung nicht vernachlässigt werden solle. Wichtige Aufgabe der Europäischen Nachbarschaftspolitik sei es, die Beziehungen mit den Nachbarländern auch jenseits des Themas ,Erweiterung' zu stärken.

Iain Begg präsentierte im Anschluss Empfehlungen für ein Gelingen der deutschen EU-

1 Vgl. Andrew Duff: Plan B: How to rescue the European Constitution, Notre Europe, Studies and Research Nr. 52, Oktober 2006. 
Ratspräsidentschaft, welche von Wissenschaftlern verschiedener Disziplinen und Länder, die im EU-CONSENT Netzwerk zusammenarbeiten, entwickelt worden sind. Das EU-CONSENT Netzwerk (Constructing Europe Network) ist ein Exzellenznetzwerk gemeinsamer Forschung und Lehre in Europa, dass die Auswirkungen von Vertiefung und Erweiterung der EU analysiert. Die Empfehlungen des Netzwerks im Einzelnen: ${ }^{2}$

- „The Constitutional Treaty which has been ratified by a majority of EU member states should be regarded as the roadmap and the basis for any further debates on institutional and political reform. Re-opening debates on institutional reform starting from the Treaty of Nice or substantially altering the contents of the Constitutional Treaty does not represent a politically acceptable alternative.

- A high level groupe de reflexion on the $\mathbf{E U}$ budget with a mandate to prepare (as the Kok report did for the re-launch of Lisbon) the 2008/9 review of the EU budget should be established, chaired by a high profile figure such as a former prime minister or person of similar standing.

- In developing the EU's energy policy, priority should be given to embedding it in a smart growth strategy which focuses on investment in sustainable energy solutions. A smart growth strategy needs to be understood not just as a contribution to sustainable development objectives, but also as a means of fostering the EU's competitive position in the global market for sustainable technologies such as wind turbines. In addition, the foreign policy and international security dimensions of energy policy choices should be highlighted.
- A common European policy on immigration should be established. Its task will be the management of immigration in a fair and sustainable way. Moreover, immigration policy will have to take into account salient issues of internal security.

- European crisis management should be made more credible, more pro-active and more effective and should be regarded as a vital instrument for expressing Europe's role and responsibility in the world. In order to do so, the civil-military cell should be reinforced in its role to make ESDP a coherent policy. More battle groups as well as more resources devoted to civilian tasks should be created, and the High Representative should be invited to the meetings of the European Commission regularly.

- The European Neighbourhood Policy needs new impetus. It is essential to include it in a coherent European Security Strategy and turn it into a credible and effective instrument of a comprehensive European foreign and security policy towards and in cooperation with the neighbours in the East and South.

- The creation of a European public space is of utmost importance and should thus explicitly be acknowledged in the Berlin Declaration. It would also be useful to explore imaginative options for creating a denser and more active European public space. These might include initiatives such as promoting the development of pan-European TV channels, or supporting the making of discussion programmes and documentaries related to European issues."

Michael Stübgen $M d B$ befasste sich vor allem mit der in Deutschland verbreiteten Erweiterungsskepsis, die von der Politik ernst genommen werden müsse. Neben der Verfassungs-

2 Die Empfehlungen basieren auf Ergebnissen des EU-CONSENT Network of Excellence. Autorinnen und Autoren: Prof. Dr. Iain Begg, London School of Economics and Political Science, London; Prof. Dr. Gianni Bonvicini, Istituto Affari Internazionali, Rom; Dr. Anne Faber, Universität Köln; Dr. Gunilla Herolf, Swedish Institute of International Affairs, Stockholm; Dr. Barbara Lippert, Institut für Europäische Politik, Berlin; Prof. Dr. Paul Luif, Österreichisches Institut für Internationale Beziehungen, Wien. 
frage seien auch die Themen Klimaschutz und Energiepolitik, einschließlich der Kontroverse über die Nutzung der Atomenergie, wichtige Punkte auf der Präsidentschaftsagenda. José Ignacio Torreblanca warnte davor, die politisch und wirtschaftlich erfolgreiche Politik der Erweiterung im Nachhinein als Versagen und Fehler umzudeuten. Deshalb sei durch eine bessere Argumentation und Kommunikation der Erweiterungsmüdigkeit entgegenzuwirken. Die schrittweise Entwicklung einer europäischen Öffentlichkeit sei unabdingbar für die Herausbildung einer europäischen Identität. So würden laut Torreblanca spanische Durschnittsbürger ohne Zögern drei bekannte Köche nennen können, doch lediglich fünf Prozent der Spanier seien in der Lage, drei Europaabgeordnete zu benennen. Maria Karasinska-Fendler betonte positive Auswirkungen der EU-Erweiterung im Hinblick auf den Demokratie-Export und die Förderung demokratischer Entwicklungen. Diese Fortschritte, die auch bei den neuen Mitgliedern der EU beobachtbar seien, sollten im Rahmen der ENP weiter ausgebaut und unterstützt werden. Gaetane Ricard-Nihoul erläuterte, dass die Erweiterung in Frankreich nie sehr populär gewesen sei, dass sich nun aber der Kontext geändert habe, da die Erweiterung der EU mit Fragen nach der Identität der EU verbunden wird. Insbesondere in der Frage um die Zukunft des VVE sei im Hinblick auf die anstehenden Präsidentschaftswahlen die Scheu groß, sich für oder gegen eine bestimmte Taktik auszusprechen.

\section{Gestaltungsraum in der europäischen Innen-} und Justizpolitik

Peter Altmaier betonte in einem Panel zum Thema „Die Sicherheit der Bürger in der EU - Leistungsmöglichkeiten der europäischen Innen- und Justizpolitik" unter dem Vorsitz von Brendan Donnelly, dass die Informationsvernetzung im Bereich der Justiz- und Innenpolitik einen Bereich darstellt, bei dem der Mehrwert der Kooperation auf EU-Ebene dem Bürger leicht vermittelbar sei. Altmaier plädierte dafür, das Schengeninformations- system (SIS) zu SIS II weiterzuentwickeln und biometrische Daten aufzunehmen. Neben dem Datenaustausch sei es notwendig, die praktische Polizeiarbeit zu verbessern. Inwieweit Polizisten aus anderen EU-Staaten hoheitliche Gewalt in einem anderen Mitgliedstaat ausüben können, müsse geklärt werden. Darüber hinaus ging er auf die Frage des Umgangs mit Migration ein. In der EU sei allgemein ein Rückgang der Asylbewerber bei gleichzeitigem Anstieg des Zustroms illegaler Einwanderer auf den Arbeitsmarkt zu beobachten. Es komme zu einer Wettbewerbsverzerrung, wenn illegale Einwanderer als billige Arbeitskräfte zur Verfügung stehen. Aus diesem Grund müsse ein europaweiter Zuwanderungs-Kontroll-Akt diskutiert werden. Generell gelte es die Ursachen für Migration in den Herkunftsländern zu bekämpfen. Perspektiven für eine legale Migration sollten in eine allgemeine Entwicklungspolitik eingebettet sein. Zur Lösung der Probleme der inneren Sicherheit beizutragen stelle auch einen praktischen Ansatzpunkt zur Überwindung der Verfassungskrise dar.

Paul Luif verwies darauf, dass die Innen- und Justizpolitik einer der dynamischsten Integrationsbereiche sei. Dennoch sei es häufig schwierig, einen Konsens in diesen Fragen herzustellen, was auf unterschiedliche Herausforderungen der einzelnen Mitgliedstaaten zurückzuführen sei. Ein möglicher Lösungsansatz für diese Problematik könne der „Vertrag von Prüm“ über die Vertiefung der grenzüberschreitenden Zusammenarbeit zur Bekämpfung des Terrorismus, der Kriminalität und der illegalen Immigration sein. Des Weiteren warf er die These auf, dass die EU nicht immer im Namen aller EU-Mitgliedstaaten auftreten müsse. Manchmal könne es sinnvoller sein, bilaterale Verträge auszuhandeln und Brüssel die Rolle eines Koordinators zukommen zu lassen. Fraglich sei zudem, ob die Implementierung des Verfassungsvertrags auf einen Schlag alles verbessern würde. Deshalb solle man sich nicht allein auf die Heilwirkung des VVE verlassen. 
Anschließend verwies Christian Ahrendt $M d B$ darauf, dass ein einheitlicher Rechtsraum Voraussetzung für eine EU-weite Verfolgung von Straftaten sei. Was bisher jedoch nicht so starke Beachtung gefunden habe sei die Situation der Beschuldigtenrechte. Prinzipiell gelte die grundsätzliche Anerkennung der Rechtssituation im Nachbarstaat, was dazu führen kann, dass ein Nachbarstaat in der EU ein Strafverfahren basierend auf einer völlig anderen Rechtsgrundlage weiterverfolgt beziehungsweise vollstreckt. Ahrendt argumentierte, dass sich die EU in diesem Bereich teilweise vom Prinzip gegenseitiger Anerkennung lösen müsse, da die Ausgestaltung innen- und rechtspolitischer Legislativakte häufig schneller vor sich gehe als die Angleichung der Rechte von Bürgern. Dies sei nicht akzeptabel.

Siegfried Magiera verwies auf Defizite der Zusammenarbeit im Rahmen der dritten Säule. Der Ratsbeschluss erfolge einstimmig, und das Europäische Parlament sei lediglich durch Anhörung beteiligt. Zudem sei die Rechtskontrolle des EuGH eingeschränkt. Dies bekomme besonderes Gewicht wenn man berücksichtigt, in welchem Spannungsverhältnis Sicherheit, Freiheit und Recht stehen können. Ersichtlich wäre dies beim Aufeinandertreffen des Informationsbedarfs der Institutionen im Bereich der Sicherheit einerseits und dem Datenschutz der Bürger andererseits. Weiter argumentierte Magiera, dass die intergouvernementale Zusammenarbeit in der dritten Säule über eine völkerrechtliche Zusammenarbeit hinausgehe, da sie Gemeinsamkeiten mit den Grundprinzipien aus der ersten Säule aufweist, wie etwa dem Kohärenzprinzip.

\section{Die Außenpolitik der EU in einer unsicheren Welt}

Das von Elfriede Regelsberger geleitete Panel zum Thema "Gemeinsame Außen- und Sicherheitspolitik in einer unsicheren Welt" wurde von Christoph Heusgen eröffnet, dem außen- und sicherheitspolitischen Berater der
Bundeskanzlerin. Er verwies darauf, dass eine sechsmonatige Ratspräsidentschaft lediglich begrenzte Möglichkeiten bietet, um Prioritäten erfolgreich zur Geltung zu bringen. Vielmehr gehe es darum, die Dinge, die auf dem Tisch liegen, abzuarbeiten beziehungsweise weiterzuverhandeln. Oberstes Ziel solle dabei sein, das Gewicht der EU zum Tragen zu bringen und mit einer Stimme zu sprechen. Aber die EU allein sei nicht fähig, die außenpolitischen Probleme zu lösen und bedarf der Kooperationspartner. Deshalb gehe es während der deutschen Ratspräsidentschaft darum, ,ideologische Schwierigkeiten“ zwischen der NATO und der EU insoweit zu überwinden, als dass dort eine Kooperation ermöglicht wird, wo sie dringend erfordert wird, insbesondere im Kosovo und in Afghanistan. Der EU-Afrika Gipfel wird während der portugiesischen Präsidentschaft stattfinden und in $\mathrm{Zu}$ sammenarbeit der , Triopräsidentschaft' mit Portugal und Slowenien vorbereitet. Auch im Rahmen der G8-Präsidentschaft wird Bundeskanzlerin Merkel einen Schwerpunkt auf Afrika legen. Zum Jahresauftakt 2007 wird die Kommission ein Energiepaket vorlegen, das Themen wie Energieeffizienz, erneuerbare Energie und Energieaußenbeziehungen behandeln wird. Der Rat wird einen ,Aktionsplan für Energie“ erarbeiten. Die Zusammenarbeit mit Russland wird hierbei von wesentlicher Bedeutung sein.

Michael Schaefer, Politischer Direktor im Auswärtigen Amt, betonte, dass die Stärkung der Sichtbarkeit des Hohen Vertreters der Gemeinsamen Außen- und Sicherheitspolitik (GASP) ein wesentliches strategisches Element der institutionellen Entwicklung der GASP sei. Das erste und dringendste Thema unter deutscher EU-Ratspräsidentschaft sei es, den Statusprozess im Kosovo voranzutreiben. Die Stabilisierung des Balkans habe eine zentrale Bedeutung für Gesamteuropa. Ziel sei es, alle Verhandlungsoptionen zu nutzen und Verzögerungen möglichst zu vermeiden, da sonst die Gefahr einer Destabilisierung des Kosovo drohen würde. Angebote müssen für alle Partner akzeptabel sein, die überwiegende Mehr- 
heit, aber auch die Minderheiten müssten Berücksichtigung finden. Der Statusprozess und dessen Stabilisierung stellen die größte zivile Mission der Europäischen Sicherheits- und Verteidigungspolitik sowie eine enorme finanzielle Herausforderung dar. Aus diesem Grund sei eine enge Abstimmung zwischen EU und NATO taktisch wie strategisch unabdingbar. Nach den Wahlen in Serbien am 21. Januar 2007 gelte es die neue, demokratisch legitimierte Regierung an die EU heranzuführen. Die Integration in EU-Strukturen beinhaltet laut Schaefer den Stabilisierungs- und Assoziierungsprozess sowie einen möglichen Kandidatenstatus, da eine klare politische Perspektive für Serbien der wichtigste Stabilitätsfaktor im Balkan sei. Dabei müsse Belgrad den Verpflichtungen des Internationalen Strafgerichtshofs nachkommen. Ausführlich behandelte Schäfer die Politik der EU gegenüber dem Iran, die die gewachsene internationale Rolle der EU belegt, und schließlich die Beziehungen zu Russland, den anderen osteuropäischen Nachbarn und den zentralasiatischen Staaten.

Geoffrey Edwards sprach sich für eine pro-aktive Außenpolitik der EU aus. Die Glaubwürdigkeit eines zivilen Europas erfordere die institutionelle Stärkung der ESVP und eine Erweiterung der politischen wie militärischen Mittel. Die systematische Beteiligung und Präsenz des Hohen Repräsentanten der GASP bei Ministertreffen könne hier als eine erste Willensbekundung verstanden werden und sei auch ohne eine institutionelle Reform möglich. Hanna Ojanen erinnerte daran, dass bisher jede Erweiterung der EU einen Ausbau der GASP nach sich gezogen habe. Graham Avery führte aus, dass zukünftige Erweiterungen der EU wesentlich langsamer stattfinden werden als in der Vergangenheit, die Beitrittsperspektive aber ein zentrales Element der EU-Außenbeziehungen und der Stabilisierungspolitik bleibe.

Ein soziales Europa - dank oder trotz der Lissabon-Strategie?

Anschließend wurden unter dem Vorsitz von Iain Begg die soziale Dimension der EU und die Lissabon-Stategie behandelt. Staatssekretär Wuermeling erläuterte, dass die Ziele „Wachstum“ und „Beschäftigung“ der Lissabon-Strategie mitunter in einem Spannungsverhältnis stehen. Ziel sollte es jedoch sein, die wirtschaftliche Dynamik Europas für mehr Wachstum und Beschäftigung zu nutzen. Der Europäische Binnenmarkt solle als ein Teil der Lösung der Globalisierungsfolgen verstanden werden. Hier müsse für Wachstum gesorgt werden, um Arbeitsplätze zu schaffen. Weiteres Ziel der deutschen Ratspräsidentschaft sei es, den Bürokratieabbau voranzutreiben, was ein Thema im Frühjahrsrat sein werde.

Staatssekretär Wasserhövel betonte, dass es im Sinne eines sozialen Europas unerlässlich sei, die Sozialpartner in einen Dialog zu involvieren und die Mitwirkung der Gewerkschaften bei der Politikgestaltung zu stärken. Ziel müsse es sein, wesentliche Elemente und Errungenschaften auch in Europa zu schützen und vergleichbare Sozialstandards in der EU herzustellen und zu wahren. Dazu seien auch internationale Vereinbarungen erforderlich. So sollten sich für Beschäftigung zuständige Minister in Europa und Asien gemeinsam über soziale Mindeststandards einigen. Die Humanisierung der Arbeitswelt und die Schaffung und Wahrung von Arbeitsplätzen, die die Menschen nicht krank machen, solle weiter voran getrieben werden. Die soziale Dimension der EU stelle ein Gebiet dar, auf dem Fortschritte dem einzelnen Menschen den Mehrwert der EU bewusst und erfahrbar werden lassen.

Angelica Schwall-Düren verwies zu Beginn darauf, dass die Gültigkeit der altbekannten Formel, wonach Wirtschaftswachstum auch zu Arbeitsplätzen führe, im Zeitalter der Globalisierung so nicht mehr ohne Einschränkung gelte. Aus diesem Grund seien Deregulierung und die Reduktion der Lasten von Unternehmen nicht mehr automatisch Mittel zur Beschäftigungsförderung. Um die Ziele von Lissabon zu erreichen sei die Förderung der Forschungs- und Bildungsinfrastruktur 
unabdingbar. Zudem müsse Steuerdumping verhindert werden. Außerdem gelte es, der Erkenntnis Rechung zu tragen, dass Wachstum auch Binnennachfrage braucht.

Willem Noë nannte als die größte Herausforderung der EU neben der Globalisierung den drohenden Bevölkerungsrückgang. Um den Anforderungen der globalisierten Arbeitswelt gerecht zu werden, müssten Arbeitnehmer immer flexibler sein. Jedoch sei es notwendig auch für soziale Sicherheit zu sorgen, etwa im Bereich der Alters- und Gesundheitsvorsorge. Ognian Hishow trat für eine kurz- und für eine langfristige Investitionsstrategie ein. Kurzfristig sollte beschäftigungsintensives Wachstum gefördert werden. Mittel- und langfristige Investitionen seien notwendig für wissensbasiertes Wachstum, da diese Investitionen bis zur Entfaltung ihrer Wirkung Zeit brauchen. Finanzielle Mittel hierfür sollten durch Kürzungen in der Landwirtschaft und durch eine Neujustierung der Strukturfonds freigesetzt werden.

\section{Neuausrichtung der EU-Nachbarschaftspoli- tik}

Das anschließende Panel „Impulse für eine neue Nachbarschaftspolitik der EU“" wurde von Gunilla Herolf geleitet. Ekkehard Brose stellte zunächst klar, dass es der Bundesregierung nicht um eine neue europäische Nachbarschaftspolitik (ENP) gehe, sondern darum, der gegenwärtigen ENP neue Impulse zu geben. Die ENP müsse sichtbarer werden, sie müsse stärkere Anreize durch substanzielle Angebote an die Nachbarn geben, helfen die Absorptionskraft der Zielländer zu verbessern und stärker nach Leistungskraft differenziert werden. Die deutsche Präsidentschaft wolle im Rahmen der ENP Osteuropa besonders betonen, da hier ein Nachholbedarf im Vergleich zum Mittelmeerraum bestehe. Die Vorschläge der Kommission zur Stärkung der ENP wolle die Bundesregierung zu konkreten Vorschlägen verdichten und am Ende ihrer Präsidentschaft als Programm vom Europäischen Rat verabschieden lassen. Als Elemente nannte Brose die Ausarbeitung eines Dialogs der Schwarzmeeranrainer, die Ausdehnung der Energiegemeinschaft Südosteuropa auf die Ukraine und Moldau sowie die Übernahme von Teilen des Acquis im Zuge der schrittweisen Integration in den Binnenmarkt.

Rutger Wissels bezeichnete die sehr begrenzte Finanzmittelausstattung sowie die Uneinigkeit der Mitgliedstaaten darüber, wie diese eingesetzt werden sollten, als grundlegende Schwierigkeit der ENP. Ein Problem der bisherigen Maßnahmen sehe er darin, dass eine Vorgehensweise allen teilnehmenden Partnerländern übergestülpt werde. Als eine bedeutende Aufgabe der ENP nannte er die Behandlung regionaler Konflikte und deren Lösung, wobei mehr Differenzierung notwendig sei. Als positives Beispiel verwies Wissels auf die Rolle der EU in Transnistrien. Ettore Greco führte aus, dass die ENP zukünftig stärker in die GASP eingebettet und Alternativen zur Beitrittsperspektive entwickelt werden sollen. Atis Lejins setzte sich kritisch mit der Russlandpolitik der EU auseinander, in deren Schatten die ENP bisweilen stehe. Krassimir Nikolov ermutigte die deutsche Präsidentschaft, das Potenzial der Kooperation in der Schwarzmeerregion zu entwickeln und zu einem festen Bestandteil der ENP zu machen. Rainder Steenblock MdB betonte die strategische Dimension der ENP, da sie an Interessen der EU in spezifischen Regionen gebunden sei. Damit komme auch ein neuer Machtanspruch und ein neues Selbstbewusstsein der EU zum Ausdruck. Steenblock forderte, für Osteuropa ein Äquivalent zum BarcelonaProzess zu entwickeln, um die vorherrschende Bilateralität zu ergänzen. Denkbar wäre zum Beispiel ein ,Riga-Prozess', wobei das Spannungsverhältnis mit den Interessen Russlands berücksichtigt werden müsse.

\section{Die Zukunft Europas}

Im Rahmen eines Abschlusspanels unter der Leitung von Christian Franck über die $\mathrm{Zu}-$ kunft Europas argumentierte Axel Schäfer $M d B$, dass ohne eine neue Verfasstheit keine 
weiteren neuen Mitglieder aufgenommen werden könnten. Die Staaten in denen die Verfassungsreferenden gescheitert sind, seien nun in der Bringschuld, um institutionelle Reformen wieder zu beginnen.

Lenka Rovna betonte die nachhaltigen Erfolge der europäischen Integration. Das vereinigte Europa verfüge über vielfältige Expertise und stelle den größten einheitlichen Binnenmarkt der Welt dar. Dies sollte sich auch durch die Ausstattung mit angemessenen politischen Werkzeugen widerspiegeln, um der eigenen Rolle gerecht zu werden. Drängende Probleme wie die demografische Entwicklung und die Frage der Energieversorgung seien Fragen, die nicht auf nationaler Ebene gelöst werden könnten. Deshalb sei eine institutionelle Reform unerlässlich, wofür Rovna den „Vertrag von Prag 2009“ als Perspektive anbot. Jaap de Zwaan betonte in seinem Beitrag die Bedeutung der Beziehungen zwischen der EU und Russland. Sie erfordere eine Abstimmung der EU im Umgang mit Themen, die einen Interessenkonflikt in sich bürgen. Alvaro de Vasconcelos warb dafür, das Nein zum VVE auch als ein Nein zu akzeptieren, da in den Referenden eine deutliche Ablehnung zum Ausdruck kam. Daraus ergebe sich aber auch eine wichtige Frage der Identität Europas. Zudem plädierte er für den Beitritt der Türkei, denn das grundlegende Element der EU sei die Vielfalt in der Einheit. Marjan Svetličič skizzierte mit der Stabilisierung des Kosovo, dem Irak-Konflikt und dem Atomkonflikt mit dem Iran die zukünftigen Herausforderungen der EU. Zudem seien die Neugestaltung der ENP und die Frage der Energieversorgung zu klären.

In seinem Schlusswort plädierte Wolfgang Wessels dafür, dass die deutsche Ratspräsidentschaft die sechs Monate nutzen sollte, um mögliche Verhandlungspakte in der Frage des Verfassungsvertrags auszuloten. Hierbei spiele auch die deutsche Position eine wichtige Rolle. Zur Aufgabe der deutschen Ratspräsidentschaft gehöre einerseits das Ausloten der Möglichkeiten, aber auch der Mut zum Risiko.

\section{Europapolitik ostdeutscher Länder}

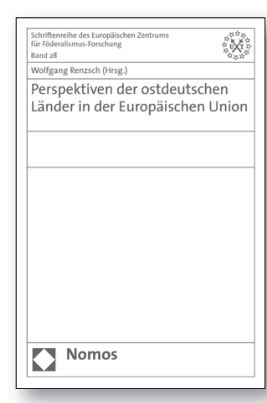

Bitte bestellen Sie bei Ihrer Buchhandlung oder bei Nomos G07221/2104-37| - 13 | www.nomos.de | sabine.horn@nomos.de mus-Forschung, Bd. 28)
Perspektiven ostdeutscher Länder in der Europäischen Union

Herausgegeben von Wolfgang Renzsch

2007, 95 S., brosch., 19,- €, ISBN 978-3-8329-2548-2

(Schriftenreihe des Europäischen Zentrums für Föderalis-

Der vorliegende Band beschäftigt sich mit den Herausforderungen und Perspektiven der ostdeutschen Bundesländer innerhalb der Europäischen Union.

Nomos 